\title{
Enantioseletive Reduction of Imines Catalyzed by a Rhenium(V)-Oxo Complex
}

\author{
Kristine A. Nolin, Richard W. Ahn, and F. Dean Toste* \\ Center for New Directions in Organic Synthesis, Department of Chemistry, \\ University of California, Berkeley, California 94720
}

\section{Supporting Information}

I. General Information $\quad 2$

II. Experimental

(a) Preparation and isolation of CN-Box 2

(b) Preparation and isolation of complex $3 \quad 3$

(c) Synthesis of phosphinyl imines 4

(d) Synthesis of $\alpha$-imino esters $\quad 5$

(e) Procedure for asymmetric reduction of imines 5

III. HMQC and HMBC spectra of $3 \quad 12$ 
General Information. Unless otherwise noted all commercial materials were purchased from Aldrich and used without purification. Small scale reactions $(<4 \mathrm{~mL})$ were carried out in Fisher Scientific disposable scintillation vials. ACS grade acetone, ethyl acetate, hexane, and dichloromethane were obtained from EM Science. Dry solvents were passed through an alumina column unless otherwise noted. $N_{, N}, N^{\prime}, N^{\prime}$ Tetramethylethylenediamine (TMEDA) was distilled from $\mathrm{CaH}$ prior to use. All reaction mixtures were stirred with a magnetic stir bar. TLC analysis of reaction mixtures was performed on Merck silica gel 60 $\mathrm{F}_{254}$ TLC plates. Chromatography was carried out on Merck 60 silica gel $(32-63 \mu \mathrm{m})$ and Aldrich neutral activated aluminum oxide ( 150 mesh, 58 $)$. ${ }^{1} \mathrm{H}$ and ${ }^{13} \mathrm{C}$ NMR spectra were recorded with Bruker AV300, AVQ-400, AVB-400, and DRX-500 spectrometers and referenced to $\mathrm{CDCl}_{3}$ unless otherwise noted. Enantiomeric excess was determined using an Agilent 1100 Series Chiral HPLC or Shimatzu 10A VP Series Chiral HPLCwith detection at $254 \mathrm{~nm}$. Mass spectral and analytical data were obtained via the Micro-Mass/Analytical Facility operated by the College of Chemistry, University of California, Berkeley. Crystal structures were solved by the Crystallographic Facilities at the University of California, Berkeley.

\section{Experimental:}

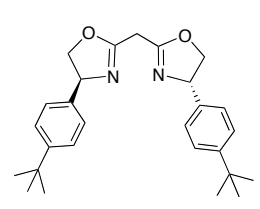

2,2'-Methylenebis[(4R)-4-(p-tButyl)phenyl-2-oxazoline.$^{1} \quad$ Diethyl malonimidate dihydrochloride $(0.962 \mathrm{mg}, 4 \mathrm{mmol})$ in dry $\mathrm{CH}_{2} \mathrm{Cl}_{2}(44 \mathrm{~mL})$ was cooled to $0{ }^{\circ} \mathrm{C}$ in an oven-dried round-bottom flask equipped with a magnetic stir bar under $\mathrm{N}_{2}$ atmosphere.

$(R$ or $S)$-2-Amino-2-(p-tert-butylphenyl)ethanol ${ }^{2}(1.9322 \mathrm{~g}, 10 \mathrm{mmol})$ was added to the reaction mixture and the ice bath was removed. The solution was stirred at room temperature for 3 days. Upon completion, the reaction was quenched with ice water and extracted three times with $\mathrm{CH}_{2} \mathrm{Cl}_{2}$. The organic phases were combined, dried with $\mathrm{Na}_{2} \mathrm{SO}_{4}$, filtered and the solvents were evaporated. The residue was chromatographed yielding a white solid. (silica gel chromatography, eluent 2.5-10\% $\mathrm{MeOH}$ in $\mathrm{CH}_{2} \mathrm{Cl}_{2}$ ); ${ }^{1} \mathrm{H}$ NMR (400 MHz, $\left.\mathrm{CDCl}_{3}\right) \delta 7.37(\mathrm{~d}, J=8.4 \mathrm{~Hz}, 4 \mathrm{H}), 7.24(\mathrm{~d}, J=8.3 \mathrm{~Hz}, 4 \mathrm{H}), 5.24(\mathrm{t}, J=9.1 \mathrm{~Hz}, 2 \mathrm{H}), 4.68$ $(\mathrm{dd}, J=10.2,8 \mathrm{~Hz}, 2 \mathrm{H}), 4.22(\mathrm{t}, J=8.1 \mathrm{~Hz}, 2 \mathrm{H}), 3.57(\mathrm{~s}, 2 \mathrm{H}), 1.31(\mathrm{~s}, 18 \mathrm{H}) ;{ }^{13} \mathrm{C}\left\{{ }^{1} \mathrm{H}\right\} \mathrm{NMR}(100 \mathrm{MHz}$, 
$\left.\mathrm{CDCl}_{3}\right) \delta$

HRMS (EI) calcd for $\left[\mathrm{C}_{27} \mathrm{H}_{34} \mathrm{~N}_{2} \mathrm{O}_{2}\right]^{+} 418.2620$, Found 418.2622. Yield: $1.3 \mathrm{~g}, 78 \%$

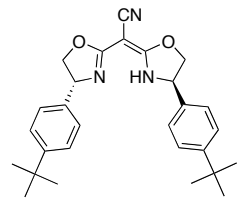

Bis-[4-(4-tert-butyl-phenyl)-4,5-dihydro-oxazol-2-yl]-acetonitrile (2). ${ }^{3}$ In an ovendried round-bottom flask, the reaction solution of bis(oxazoline) $(1.3063 \mathrm{~g}, 3.12 \mathrm{mmol})$ in dry THF $(16 \mathrm{~mL})$ was cooled to $-78{ }^{\circ} \mathrm{C} . n \mathrm{BuLi}, 1.38 \mathrm{~mL}(2.5 \mathrm{M}$ in hexanes), was added slowly to the reaction solution. Immediately following, TMEDA ( $0.55 \mathrm{~mL}, 3.64 \mathrm{mmol})$ was added to the reaction solution and the solution was stirred at $-78^{\circ} \mathrm{C}$ for $1 \mathrm{~h}$ then $0{ }^{\circ} \mathrm{C}$ for $30 \mathrm{~min}$. The reaction solution was cooled to $-78{ }^{\circ} \mathrm{C}$ and 1.12 equiv of $p$-toluenesulfonyl cyanide was added. The reaction solution was allowed to warm slowly to room temperature and stirred overnight. After $12 \mathrm{~h}$, the reaction was quenched with sat. $\mathrm{NH}_{4} \mathrm{Cl}$ and was stirred at room temperature for 5 min then triturated with $\mathrm{Et}_{2} \mathrm{O}$ and filtered. The reaction solution was extracted 3 times with $\mathrm{Et}_{2} \mathrm{O}$ and 2 times with $\mathrm{CH}_{2} \mathrm{Cl}_{2}$. The organic layers were combined, dried, filtered, and the solvents were evaporated. The residue was then chromatographed yielding a white solid, the pure cyanobis(oxazoline), $925 \mathrm{mg}, 67 \%$ (neutral activated aluminum oxide chromatography, eluent $20 \%$ EtOAc in hexanes); ${ }^{1} \mathrm{H}$ NMR $\left(400 \mathrm{MHz}, \mathrm{CDCl}_{3}\right) \delta 7.38(\mathrm{~d}, J$ $=8.3 \mathrm{~Hz}, 4 \mathrm{H}), 7.17(\mathrm{~d}, J=8.3 \mathrm{~Hz}, 4 \mathrm{H}), 5.14-5.10(\mathrm{~m}, 2 \mathrm{H}), 4.83-4.78(\mathrm{~m}, 2 \mathrm{H}), 4.33-4.29(\mathrm{~m}, 2 \mathrm{H}), 1.30(\mathrm{~s}$, $18 \mathrm{H}) ;{ }^{13} \mathrm{C}\left\{{ }^{1} \mathrm{H}\right\} \mathrm{NMR}\left(100 \mathrm{MHz}, \mathrm{CDCl}_{3}\right) \delta 167.6,151.6,137.6,126.2,126.0,76.0,64.2,34.6,31.3 ;$ Anal. Calcd. for $\mathrm{C}_{28} \mathrm{H}_{33} \mathrm{~N}_{3} \mathrm{O}_{2}$ : C 75.81, H 7.50, N 9.47; Found C 75.64, H 7.61, N 9.20.

General Procedure for Synthesis of Catalyst 3. $\operatorname{Re}(\mathrm{V})$ dimethylsulfide complex $\mathbf{1}^{4}$ (1 equiv) was added to a round-bottom flask charged with a magnetic stir bar. $\mathrm{CH}_{2} \mathrm{Cl}_{2}$ was added to a reaction flask to a concentration of $5 \mathrm{mM}$. In a scintillation vial, 1.1 equiv of cyanobis(oxazoline) ligand was diluted in $\mathrm{CH}_{2} \mathrm{Cl}_{2}$ (5 mM). Ligand solution was added slowly to Re suspension. Reaction solution quickly changes from light green to very dark green. Reaction stirred at $\mathrm{rt}$ for $\sim 5 \mathrm{~h}$ at which time the solution is a bright emerald green. (Note: This reaction time can be reduced to 30 minutes by adding 1 drop of DMSO; however, isolation of the product becomes more difficult.) Solvent was evaporated and the dark green film was diluted with a small amount of $\mathrm{CH}_{2} \mathrm{Cl}_{2}$ then triturated with $\mathrm{Et}_{2} \mathrm{O}$. Filtration with a Buchner funnel 
yields Re complex 3, a bright green solid. The trituration was repeated 4 times or until no significant material was isolated. The resultant solid was dried in vacuo.

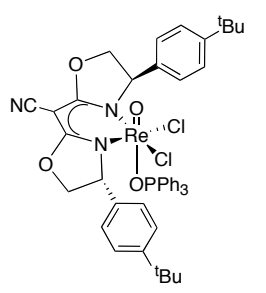

$\operatorname{Re}(\mathbf{V})$ complex 3: Yield: $74 \% ;{ }^{1} \mathrm{H}$ NMR $\left(400 \mathrm{MHz}, \mathrm{CD}_{2} \mathrm{Cl}_{2}\right) \delta 7.65(\mathrm{t}, J=7.6$ $\mathrm{Hz}, 3 \mathrm{H}), 7.52-7.47(\mathrm{~m}, 6 \mathrm{H}), 7.28-7.15(\mathrm{~m}, 8 \mathrm{H}), 6.95(\mathrm{dd}, J=7.6,2.1 \mathrm{~Hz}, 4 \mathrm{H}), 6.62(\mathrm{bs}$, $2 \mathrm{H}), 6.06(\mathrm{~d}, J=6.4 \mathrm{~Hz}, 1 \mathrm{H}), 5.19(\mathrm{bs}, 1 \mathrm{H}), 4.81(\mathrm{t}, J=8.4 \mathrm{~Hz}, 1 \mathrm{H}), 4.64(\mathrm{~d}, J=8.4$ $\mathrm{Hz}, 1 \mathrm{H}), 4.46(\mathrm{~d}, J=6.4 \mathrm{~Hz}, 1 \mathrm{H}), 4.19$ (bs, $1 \mathrm{H}), 1.24(\mathrm{~s}, 9 \mathrm{H}), 0.94(\mathrm{~s}, 9 \mathrm{H}) ;{ }^{13} \mathrm{C}\left\{{ }^{1} \mathrm{H}\right\}$ NMR Dept $135\left(100 \mathrm{MHz}, \mathrm{CD}_{2} \mathrm{Cl}_{2}\right) \delta 135.4,130.7,130.5,128.9 ;{ }^{31} \mathrm{P}\left\{{ }^{1} \mathrm{H}\right\}\left(162 \mathrm{MHz}, \mathrm{CD}_{2} \mathrm{Cl}_{2}\right) 43.2$ (very broad, s); IR 2950, 2215, 1600, 1493, 1135, 1086, $996 v$ max cm${ }^{-1}$; Anal. Calcd. for $\mathrm{C}_{46} \mathrm{H}_{47} \mathrm{Cl}_{2} \mathrm{~N}_{3} \mathrm{O}_{4} \mathrm{PRe}: \mathrm{C}$ 55.58, H 4.77, N 4.23; Found C 55.31, H 5.09, N 3.98.

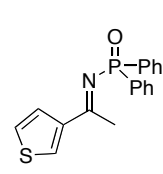

Imine 4 was synthesized by modification literature procedure. ${ }^{5}$ This procedure was followed for the synthesis of imines $\mathbf{6 a - j}$ and 10a-f. Oxime $(12.6 \mathrm{mmol})$ was added to an oven-dried round-bottom flask charged with a magnetic stir bar under $\mathrm{N}_{2}$. Dry $\mathrm{CH}_{2} \mathrm{Cl}_{2}(150 \mathrm{~mL})$ and hexanes $(150 \mathrm{~mL})$ were added to a reaction flask. Dry triethylamine $(2.00 \mathrm{~mL}, 14.2 \mathrm{mmol})$ was added to the reaction solution, which was then cooled to $-45^{\circ} \mathrm{C}$ and stirred for 5 minutes. Chlorodiphenylphosphine (2.54 mL, $14.1 \mathrm{mmol}$ ) was diluted with $10 \mathrm{~mL}$ of dry $\mathrm{CH}_{2} \mathrm{Cl}_{2}$ and then was added dropwise to reaction solution over a period of 45 minutes. The reaction solution was stirred at $-45^{\circ} \mathrm{C}$ for 1 hour, slowly warmed to room temperature, and then stirred for 1 hour at room temperature. The solvents were evaporated under $20^{\circ} \mathrm{C}$. The residue was diluted $\mathrm{CH}_{2} \mathrm{Cl}_{2}$ and washed twice with water and once with brine. The organic phase was dried with $\mathrm{Na}_{2} \mathrm{SO}_{4}$, filtered, and solvents evaporated. The residue was chromatographed yielding the phosphinyl imine (silica gel chromatography, eluent $10-50 \%$ acetone in $\mathrm{CH}_{2} \mathrm{Cl}_{2}$ ). (On occasion, recrystallization was needed for isolation of pure imine.) Yield: $61 \%$; ${ }^{1} \mathrm{H}$ NMR (400 MHz, $\left.\mathrm{CDCl}_{3}\right) \delta 8.03-7.98(\mathrm{~m}, 4 \mathrm{H}), 7.62(\mathrm{~d}, J=3.6 \mathrm{~Hz}, 1 \mathrm{H}), 7.58(\mathrm{~d}, J=4.8 \mathrm{~Hz}, 1 \mathrm{H}), 7.45-7.41(\mathrm{~m}, 6 \mathrm{H}), 7.10(\mathrm{t}, J$ $=4.4 \mathrm{~Hz}, 1 \mathrm{H}), 2.90(\mathrm{~d}, J=1.6 \mathrm{~Hz}, 3 \mathrm{H}) ;{ }^{13} \mathrm{C}\left\{{ }^{1} \mathrm{H}\right\} \quad \mathrm{NMR}\left(100 \mathrm{MHz}, \mathrm{CDCl}_{3}\right) \delta 174.7(\mathrm{~d}, J=5.8 \mathrm{~Hz}), 147.6$ $(\mathrm{d}, J=27.4 \mathrm{~Hz}), 135.6,134.3,133.5,131.6(\mathrm{~d}, J=9.0 \mathrm{~Hz}), 131.4(\mathrm{~d}, J=2.4 \mathrm{~Hz}), 128.5(\mathrm{~d}, J=12.4 \mathrm{~Hz})$, 128.5, $22.9(\mathrm{~d}, J=11.0 \mathrm{~Hz}) ;{ }^{31} \mathrm{P}$ NMR $\left(162 \mathrm{MHz}, \mathrm{CDCl}_{3}\right) \delta 18.3$. HRMS (EI) calcd for $\left[\mathrm{C}_{18} \mathrm{H}_{16} \mathrm{NOPS}\right]^{+}$ 
325.0690, Found 325.0688; Anal. Calcd. for $\mathrm{C}_{18} \mathrm{H}_{16} \mathrm{NOPS}$ : C 66.45, H 4.96, N 4.30, S 9.86; Found C 66.29, H 5.14, N 4.20, S 9.72.

for the synthesis of imines $\mathbf{8}$ b-c. Imine was purified by column chromatography (silica gel chromatography, eluent $10-50 \%$ acetone in $\left.\mathrm{CH}_{2} \mathrm{Cl}_{2}\right)$. Yield: $61 \% ;{ }^{1} \mathrm{H} \mathrm{NMR}\left(400 \mathrm{MHz}, \mathrm{CDCl}_{3}\right) \delta 7-99-$ $7.90(\mathrm{~m}, 6 \mathrm{H}), 7.59-7.55(\mathrm{~m}, 1 \mathrm{H}), 7.49-7.41(\mathrm{~m}, 8 \mathrm{H}), 4.55(\mathrm{q}, J=7.2 \mathrm{~Hz}, 2 \mathrm{H}), 1.40(\mathrm{t}, J=7.2 \mathrm{~Hz}, 3 \mathrm{H})$; ${ }^{13} \mathrm{C}\left\{{ }^{1} \mathrm{H}\right\} \operatorname{NMR}\left(100 \mathrm{MHz}, \mathrm{CDCl}_{3}\right) \delta 170.5(\mathrm{~d}, J=8.0 \mathrm{~Hz}), 165.6(\mathrm{~d}, J=16 \mathrm{~Hz}), 133.8,133.6,133.3,132.0-$ $131.6(\mathrm{~m}), 129.9(\mathrm{~d}, J=13.0 \mathrm{~Hz}), 128.5(\mathrm{~d}, J=13 \mathrm{~Hz}), 62.6,14.0 ;{ }^{31} \mathrm{P} \mathrm{NMR}\left(162 \mathrm{MHz}, \mathrm{CDCl}_{3}\right) \delta 21.7$. HRMS (EI) calcd for $\left[\mathrm{C}_{22} \mathrm{H}_{21} \mathrm{NO}_{3} \mathrm{P}\right]^{+}$378.1259, Found 378.1259; Anal. Calcd. for $\mathrm{C}_{18} \mathrm{H}_{16} \mathrm{NOPS}$ : C 70.02, H 5.34, N 3.71; Found C 69.81, H 5.18, N 3.55.

General Procedure for Asymmetric Reduction of Phosphinyl Imines with Asymmetric Catalyst. To a $7.4 \mathrm{~mL}$ Fischer vial charged with a solution of imine (50 mg, 1 equiv) in $\mathrm{CH}_{2} \mathrm{Cl}_{2}(1 \mathrm{M})$, was added silane (2 equiv) followed by catalyst 1 (3 mol\%). The reaction was monitored by TLC. Upon completion or 72 hours, the reaction was chromatographed. The reaction mixture was loaded directly on to a silica gel column and chromatographed with the $10-50 \%$ acetone in $\mathrm{CH}_{2} \mathrm{Cl}_{2}$ to give the amine. Absolute configurations were assigned by comparison of the HPLC retention times to literature values. ${ }^{7}$

$7.20(\mathrm{~m}, 1 \mathrm{H}), 6.94-6.92(\mathrm{~m}, 2 \mathrm{H}), 4.62-4.53(\mathrm{~m}, 1 \mathrm{H}), 3.36-3.32(\mathrm{~m}, 1 \mathrm{H}), 1.70(\mathrm{~d}, J=6.8 \mathrm{~Hz}$, $3 \mathrm{H}) ;{ }^{13} \mathrm{C}\left\{{ }^{1} \mathrm{H}\right\}$ NMR $\left(100 \mathrm{MHz}, \mathrm{CDCl}_{3}\right) \delta 149.7(\mathrm{~d}, J=9.0 \mathrm{~Hz}), 133.5,132.5,132.5(\mathrm{~d}, J=9.5 \mathrm{~Hz}), 132.3$, 132.2, 131.9, 131.8, 131.0, 128.5 (d, $J=12.5 \mathrm{~Hz}), 126.9,124.1,123.6,47.2,26.0 ;{ }^{31} \mathrm{P} \mathrm{NMR}(162 \mathrm{MHz}$, $\left.\mathrm{CDCl}_{3}\right) \delta$ 22.1; HRMS (EI) calcd for $\left[\mathrm{C}_{18} \mathrm{H}_{19} \mathrm{NOPS}\right]^{+}$328.0925, Found 328.0929; Anal. Calcd. for $\mathrm{C}_{18} \mathrm{H}_{18}$ NOPS: C 66.04, H 5.54, N 4.28, S 9.79; Found C 65.74, H 5.70, N 4.32, S 9.42; \%ee by HPLC (Chiralcel OD Column , 2-propanol:hexanes $=10: 90(1.0 \mathrm{~mL} / \mathrm{min})$, minor isomer $8.3 \mathrm{~min}$, major isomer 10.2min): $99 \%$ ee. 
$\mathrm{HNN}^{-\mathrm{P}(\mathrm{O}) \mathrm{Ph}_{2}}$ (7a) Yield: 51\%; \% \%ee by HPLC (Chiralcel OD Column , 2-propanol:hexanes = 10:90 (1.0 mL/min), minor isomer $7.9 \mathrm{~min}$, major isomer $10.5 \mathrm{~min}): 99 \%$ ee.

$6 \mathrm{H}), 7.21(\mathrm{~d}, J=9.0 \mathrm{~Hz}, 2 \mathrm{H}), 6.84(\mathrm{~d}, J=9.0 \mathrm{~Hz}, 2 \mathrm{H}), 4.40-4.30(\mathrm{~m}, 1 \mathrm{H}), 3.79(\mathrm{~s}$, $3 \mathrm{H}), 3.23-3.13(\mathrm{~m}, 1 \mathrm{H}), 1.55(\mathrm{~d}, J=6.5 \mathrm{~Hz}, 3 \mathrm{H}) ;{ }^{13} \mathrm{C}\left\{{ }^{1} \mathrm{H}\right\} \quad \mathrm{NMR}\left(100 \mathrm{MHz}, \mathrm{CDCl}_{3}\right) \delta 158.6,133.6,132.4-$ 131.6 (m), 128.5-128.3 (m), 127.1, 113.8, 55.2, 50.4, 25.8; ${ }^{31} \mathrm{P}$ NMR (162 MHz, $\left.\mathrm{CDCl}_{3}\right) \delta 21.3$; HRMS (EI) calcd for $\left[\mathrm{C}_{21} \mathrm{H}_{23} \mathrm{NO}_{2} \mathrm{P}\right]^{+} 352.1466$, Found 352.1468; \%ee by HPLC (Chiralcel OD Column , 2propanol:hexanes = 10:90 (1.0 mL/min), minor isomer $10.3 \mathrm{~min}$, major isomer $11.5 \mathrm{~min}): 98 \%$ ee.

7.55-7.33(m,10H), 4.49-4.38(m,1H), 3.38-3.34(m, 1H), $1.58(\mathrm{~d}, J=6.8 \mathrm{~Hz}, 3 \mathrm{H})$; ${ }^{13} \mathrm{C}\left\{{ }^{1} \mathrm{H}\right\} \quad \mathrm{NMR}\left(100 \mathrm{MHz}, \mathrm{CDCl}_{3}\right) \delta 149.0(\mathrm{~d}, J=9 \mathrm{~Hz}), 132.4-131.9(\mathrm{~m}), 128.7-128.4(\mathrm{~m}), 126.4,125.5$ $(\mathrm{bq}, \mathrm{J}=4 \mathrm{~Hz}), 50.7,25.8(\mathrm{~d}, J=4 \mathrm{~Hz}) ;{ }^{19} \mathrm{~F}$ NMR $\left(376 \mathrm{MHz}, \mathrm{CDCl}_{3}\right) \delta-61.63 ;{ }^{31} \mathrm{P} \mathrm{NMR}(162 \mathrm{MHz}$ $\left.\mathrm{CDCl}_{3}\right) \delta$ 22.9; HRMS (EI) calcd for $\left[\mathrm{C}_{21} \mathrm{H}_{20} \mathrm{~F}_{3} \mathrm{NOP}\right]^{+}$390.1235, Found 390.1233; Anal. Calcd. for $\mathrm{C}_{18} \mathrm{H}_{18}$ NOPS: C 64.78, H 4.92, N 3.60; Found C 64.55, H 5.13, N 3.43; \%ee by HPLC (Chiralcel OD Column , 2-propanol:hexanes = 10:90 (1.0 mL/min), minor isomer $7.7 \mathrm{~min}$, major isomer $9.1 \mathrm{~min}): 98 \%$ ee.

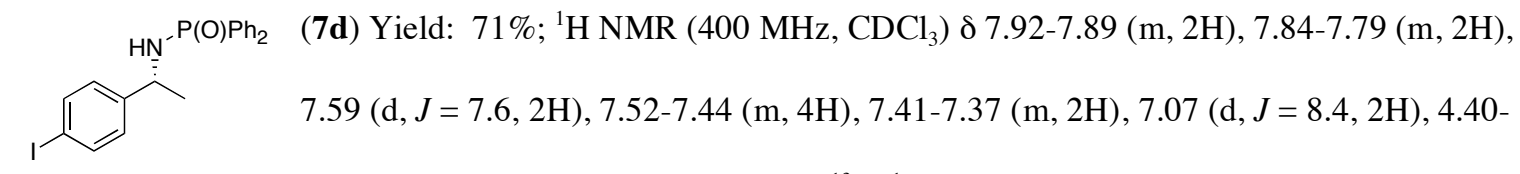
$4.30(\mathrm{~m}, 1 \mathrm{H}), 3.37-3.33(\mathrm{~m}, 1 \mathrm{H}), 1.57(\mathrm{~d}, J=6.8 \mathrm{~Hz}, 3 \mathrm{H}) ;{ }^{13} \mathrm{C}\left\{{ }^{1} \mathrm{H}\right\}$ NMR $\left(100 \mathrm{MHz}, \mathrm{CDCl}_{3}\right) \delta 144.8(\mathrm{~d}, J$ $=6 \mathrm{~Hz}), 137.6,132.7-131.9(\mathrm{~m}), 128.6-128.4(\mathrm{~m}), 128.2,92.4,50.6,25.8(\mathrm{~d}, J=3 \mathrm{~Hz}) ;{ }^{31} \mathrm{P}$ NMR $(162$ $\left.\mathrm{MHz}, \mathrm{CDCl}_{3}\right) \delta 22.9$; HRMS (EI) calcd for $\left[\mathrm{C}_{20} \mathrm{H}_{20} \mathrm{INOP}\right]^{+}$448.0327, Found 488.0336; \%ee by HPLC (Chiralcel OD Column, ethanol:hexanes = 2:98 $(1.5 \mathrm{~mL} / \mathrm{min})$, minor isomer $12.5 \mathrm{~min}$, major isomer, 14.3 min): $99 \%$ ee. 


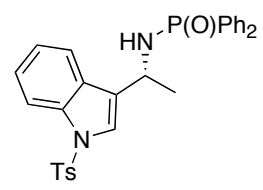

(7e) Yield: $81 \%$; ${ }^{1} \mathrm{H}$ NMR $\left(400 \mathrm{MHz}, \mathrm{CDCl}_{3}\right) \delta 8.00-7.78(\mathrm{~m}, 7 \mathrm{H}), 7.63(\mathrm{~d}, J=7.8 \mathrm{~Hz}$, 1H), 7.56-7.47 (m, 5H), 7.39-7.23 (m, 6H), 4.74-4.62 (m, 1H), 3.35-3.26 (m, 1H), 2.38 (s, 3H), $1.68(\mathrm{~d}, J=6.4 \mathrm{~Hz}, 3 \mathrm{H}) ;{ }^{13} \mathrm{C}\left\{{ }^{1} \mathrm{H}\right\} \quad \mathrm{NMR}\left(100 \mathrm{MHz}, \mathrm{CDCl}_{3}\right) \delta 145.0,135.4(\mathrm{~d}$, $J=22 \mathrm{~Hz}), 132.2-131.9(\mathrm{~m}), 130.0,128.7-128.4(\mathrm{~m}), 126.9,124.9,123.2,122.6,120.4,113.7,43.9,24.6$, 21.6; ${ }^{31} \mathrm{P}$ NMR $\left(162 \mathrm{MHz}, \mathrm{CDCl}_{3}\right) \delta 23.08$; HRMS (EI) calcd for $\left[\mathrm{C}_{29} \mathrm{H}_{27} \mathrm{~N}_{2} \mathrm{O}_{3} \mathrm{PS}\right]^{+}$515.1558, Found 515.1556; Anal. Calcd. for $\mathrm{C}_{29} \mathrm{H}_{27} \mathrm{~N}_{2} \mathrm{O}_{3}$ PS: C 67.69, H 5.27, N 5.44, S 6.23; Found C 67.30, H 5.27, N 5.32, S 6.40; \%ee by HPLC (Chiralcel OD Column , 2-propanol:hexanes $=10: 90(1.0 \mathrm{~mL} / \mathrm{min})$, minor isomer $29.0 \mathrm{~min}$, major isomer $40.7 \mathrm{~min}$ ): $99 \%$ ee.

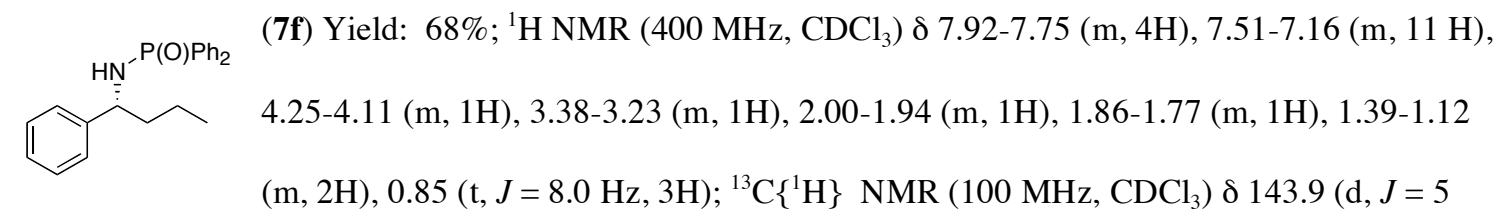

$\mathrm{Hz}), 133.9-131.6(\mathrm{~m}), 128.5-128.2(\mathrm{~m}), 127.0,126.4,55.6,42.0,19.4,13.8 ;{ }^{31} \mathrm{P} \mathrm{NMR}\left(162 \mathrm{MHz}, \mathrm{CDCl}_{3}\right) \delta$ 22.6; HRMS (EI) calcd for $\left[\mathrm{C}_{22} \mathrm{H}_{25} \mathrm{NOP}\right]^{+} 350.1674$, Found 350.1666; Anal. Calcd. for $\mathrm{C}_{22} \mathrm{H}_{24} \mathrm{NOP}$ : C 75.62, H 6.92, N 4.01; Found C 75.46, H 7.09, N 3.87; \%ee by HPLC (Chiralcel OD Column , 2 propanol:hexanes $=10: 90(1.0 \mathrm{~mL} / \mathrm{min})$, minor isomer $6.3 \mathrm{~min}$, major isomer $10.2 \mathrm{~min}): 99 \%$ ee.

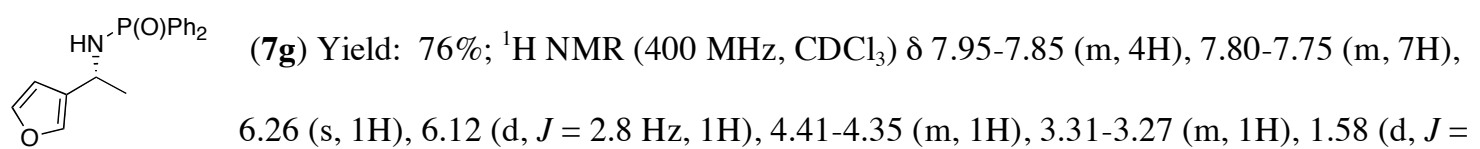
$6.8 \mathrm{~Hz}, 3 \mathrm{H}) ;{ }^{13} \mathrm{C}\left\{{ }^{1} \mathrm{H}\right\} \quad \mathrm{NMR}\left(100 \mathrm{MHz}, \mathrm{CDCl}_{3}\right) \delta 157.0(\mathrm{~d}, J=8 \mathrm{~Hz}), 141.7,133.6-131.2(\mathrm{~m}), 128.5$, 128.4, 110.1, 105.2, 45.1, 23.1; ${ }^{31} \mathrm{P}$ NMR (162 MHz, $\left.\mathrm{CDCl}_{3}\right) \delta 22.5 ; \mathrm{HRMS}$ (EI) calcd for $\left[\mathrm{C}_{18} \mathrm{H}_{18} \mathrm{NO}_{2} \mathrm{P}\right]^{+}$ 311.1075, Found 311.1072; Anal. Calcd. for $\mathrm{C}_{18} \mathrm{H}_{18} \mathrm{NO}_{2} \mathrm{P}: \mathrm{C} 69.45, \mathrm{H} 5.83, \mathrm{~N} 4.50$; Found C 69.22, $\mathrm{H}$ 5.81, N 4.67; \%ee by HPLC (Chiralcel OD Column , 2-propanol:hexanes $=5: 95(1.0 \mathrm{~mL} / \mathrm{min})$, major isomer $12.2 \mathrm{~min}$, minor isomer $13.7 \mathrm{~min}$ ): $99 \%$ ee.

\footnotetext{
$\mathrm{HN}^{\mathrm{P}(0) \mathrm{Ph}_{2}}$ (7h) Yield: 71\%;7 \%ee by HPLC (Chiralcel OD Column , 2-propanol:hexanes $=10: 90$ (1.0 mL/min), minor isomer $7.13 \mathrm{~min}$, minor isomer $12.6 \mathrm{~min})$ : $96 \%$ ee.
} 
$\mathrm{HN}^{-\mathrm{P}(\mathrm{O}) \mathrm{Ph}_{2}}$ (7i) Yield: 89\%; \% \%ee by HPLC (Chiralcel OD Column, 2-propanol:hexanes = 5:95 (1.0

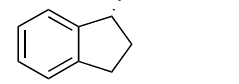
$\mathrm{mL} / \mathrm{min}$ ), minor isomer $12.2 \mathrm{~min}$, major isomer $13.7 \mathrm{~min}$ ): $95 \%$ ee.

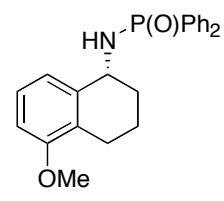

(7j) Yield: $69 \%$; ${ }^{1} \mathrm{H}$ NMR $\left(400 \mathrm{MHz}, \mathrm{CDCl}_{3}\right) \delta 8.04-8.00(\mathrm{~m}, 4 \mathrm{H}), 7.54-7.50(\mathrm{~m}, 6 \mathrm{H})$, $7.37(\mathrm{~d}, J=7.6 \mathrm{~Hz}, 1 \mathrm{H}) 7.24(\mathrm{t}, J=8.0 \mathrm{~Hz}, 1 \mathrm{H}), 6.76(\mathrm{~d}, J=8.0 \mathrm{~Hz}), 4.40-4.31(\mathrm{~m}, 1 \mathrm{H})$, $3.83(\mathrm{~s}, 3 \mathrm{H}), 3.30-3.22(\mathrm{~m}, 1 \mathrm{H}), 2.69-2.55(\mathrm{~m}, 2 \mathrm{H}), 2.14-2.05(\mathrm{~m}, 1 \mathrm{H}), 2.00-1.83(\mathrm{~m}, 2 \mathrm{H})$, 1.81-1.70 (m, 1H); ${ }^{13} \mathrm{C}\left\{{ }^{1} \mathrm{H}\right\}$ NMR (100 MHz, $\left.\mathrm{CDCl}_{3}\right) \delta$ 157.0, 139.8, 132.4-131.9 (m), 128.7-128.5 (m), 126.5, 121.0, 108.2, 55.37, 49.8, 32.7, 22.91, 19.1; ${ }^{31} \mathrm{P}$ NMR (162 MHz, $\left.\mathrm{CDCl}_{3}\right) \delta 22.1 ; \mathrm{HRMS}$ (EI) calcd for $\left[\mathrm{C}_{23} \mathrm{H}_{25} \mathrm{NO}_{2} \mathrm{P}\right]^{+}$378.1623, Found 378.1630; \%ee by HPLC (Chiralcel OD Column , 2-propanol:hexanes = 10:90 $(1.0 \mathrm{~mL} / \mathrm{min})$, major isomer $10.7 \mathrm{~min}$, minor isomer $12.2 \mathrm{~min}): 99 \%$ ee.

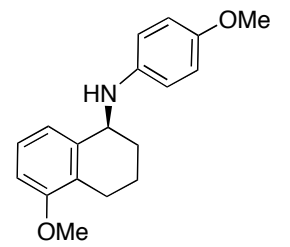

(7k) Yield: $76 \% ;{ }^{1} \mathrm{H}$ NMR $\left(400 \mathrm{MHz}, \mathrm{CDCl}_{3}\right) \delta 7.14(\mathrm{t}, J=8 \mathrm{~Hz}, 1 \mathrm{H}), 7.04(\mathrm{~d}, J=8$ $\mathrm{Hz}, 1 \mathrm{H}), 6.80(\mathrm{~d}, J=8.8 \mathrm{~Hz}, 2 \mathrm{H}), 6.74(\mathrm{~d}, J=8 \mathrm{~Hz}, 1 \mathrm{H}), 6.63(\mathrm{~d}, J=8 \mathrm{~Hz}, 2 \mathrm{H}), 4.25$ (t, $J=4.0 \mathrm{~Hz}, 1 \mathrm{H}), 3.83(\mathrm{~s}, 3 \mathrm{H}), 3.76(\mathrm{~s}, 3 \mathrm{H}), 3.60(\mathrm{bs}, 1 \mathrm{H}), 2.81-2.74(\mathrm{~m}, 1 \mathrm{H}), 2.59-$ $2.51(\mathrm{~m}, 1 \mathrm{H}), 1.97-1.71(\mathrm{~m}, 4 \mathrm{H}) ;{ }^{13} \mathrm{C}\left\{{ }^{1} \mathrm{H}\right\} \quad \mathrm{NMR}\left(100 \mathrm{MHz}, \mathrm{CDCl}_{3}\right) \delta$ 157.0, 151.8, 141.8, 139.6, 126.6, 126.2, 121.2, 115.0, 114.2, 108.0, 55.8, 55.3, 52.0, 28.1, 23.0, 18.6; HRMS (EI) calcd for $\left[\mathrm{C}_{18} \mathrm{H}_{21} \mathrm{NO}_{2}\right]^{+}$ 283.1572, Found 283.1574; \%ee by HPLC (Chiralcel OD Column , 2-propanol:hexanes = 1:99 (1.0 $\mathrm{mL} / \mathrm{min}$ ), major isomer $18.1 \mathrm{~min}$, minor isomer $20.3 \mathrm{~min}$ ): $92 \%$ ee.

$\mathrm{HN}-\mathrm{P}(0) \mathrm{Ph}_{2}$ (7l) Yield: $69 \% ;{ }^{1} \mathrm{H}$ NMR $\left(400 \mathrm{MHz}, \mathrm{CDCl}_{3}\right) \delta$ 7.93-7.86 (m, 4H), 7.48-7.38 (m, 6H), Oن 3.06-2.94 (m, 1H), 2.78-2.75 (m, 1H), 1.78-1.68 (m, 3H), 1.67-1.58 (m, 2H), 1.41-1.31 (m, 1H), 1.26-0.87 (m, 5H), $1.16(\mathrm{~d}, J=6.8 \mathrm{~Hz}, 3 \mathrm{H}) ;{ }^{13} \mathrm{C}\left\{{ }^{1} \mathrm{H}\right\} \quad \mathrm{NMR}\left(100 \mathrm{MHz}, \mathrm{CDCl}_{3}\right) \delta$ 134.1, 133.6, 132.8, 132.3-132.1 (m), 131.9-131.7 (m), 128.5-128.3 (m), 52.0 (d, $J=2.0 \mathrm{~Hz}), 45.0$ (d, $J=6 \mathrm{~Hz}), 29.4$, 28.3, 26.5, 26.4, 26.3, $20.6(\mathrm{~d}, J=4 \mathrm{~Hz}) ;{ }^{31} \mathrm{P}$ NMR $\left(162 \mathrm{MHz}, \mathrm{CDCl}_{3}\right) \delta 22.1$; HRMS (EI) calcd for $\left[\mathrm{C}_{20} \mathrm{H}_{27} \mathrm{NOP}\right]^{+}$328.1830, Found 328.1829; Anal. Calcd. for $\mathrm{C}_{20} \mathrm{H}_{26} \mathrm{NOP}$ : C 73.37, H 8.00, N 4.28; Found C 72.37, H 7.90, N 4.15. \%ee by HPLC (Chiralcel OD Column , ethanol:hexanes $=1: 99(1.0 \mathrm{~mL} / \mathrm{min})$, major isomer $10.2 \mathrm{~min}$, minor isomer $13.0 \mathrm{~min}$ ): $32 \%$ ee. 


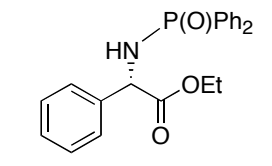

(9a) Yield: $83 \% ;{ }^{1} \mathrm{H}$ NMR (400 MHz, $\left.\mathrm{CDCl}_{3}\right) \delta$ 7.89-7.86 (m, 2H), 7.76-7.62 (m, 3H), 7.53-7.38 (m, 5H), 7.35-7.22 (m, 5H), $4.91(\mathrm{t}, J=9.9 \mathrm{~Hz}, 1 \mathrm{H}), 4.35-4.30(\mathrm{~m}, 1 \mathrm{H}), 4.23-$ $4.02(\mathrm{~m}, 2 \mathrm{H}), 1.14(\mathrm{t}, J=7.2 \mathrm{~Hz}, 3 \mathrm{H}) ;{ }^{13} \mathrm{C}\left\{{ }^{1} \mathrm{H}\right\} \mathrm{NMR}\left(100 \mathrm{MHz}, \mathrm{CDCl}_{3}\right) \delta 172.4,138.6(\mathrm{~d}, J=8 \mathrm{~Hz})$, 133.2, 132.7-132.1 (m), 131.5, 130.85, 129.0-128.4 (m), 127.3, 62.3, 57.2, 14.22; ${ }^{31} \mathrm{P}$ NMR (162 MHz, $\left.\mathrm{CDCl}_{3}\right) \delta$ 23.9; HRMS (EI) calcd for $\left[\mathrm{C}_{19} \mathrm{H}_{17} \mathrm{NOP}\right]^{+}(\mathrm{M}-\bullet \mathrm{C}(\mathrm{O}) \mathrm{OEt}) 306.1049$, Found 306.1052; Anal. Calcd. for $\mathrm{C}_{22} \mathrm{H}_{20} \mathrm{NO}_{3} \mathrm{P}$ : C 69.65, H 5.84, N 3.69; Found C 69.44, H 5.84, N 3.47; \%ee by HPLC (Chiralcel OJ Column , 2-propanol:hexanes = 5:95 (1.0 mL/min), major isomer $12.1 \mathrm{~min}$, minor isomer $22.5 \mathrm{~min}): 99 \%$ ee.

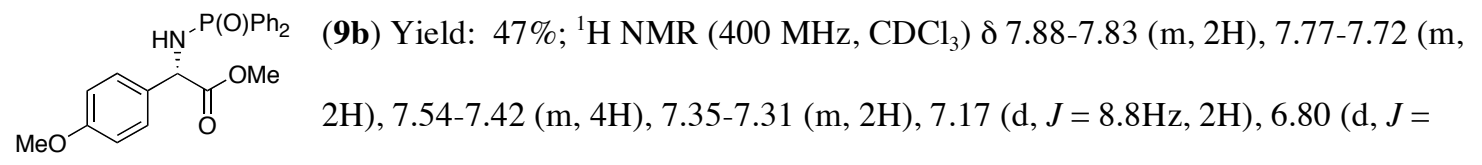
$8.8 \mathrm{~Hz}, 2 \mathrm{H}), 4.90(\mathrm{t}, J=10.0 \mathrm{~Hz}, 1 \mathrm{H}), 4.29-4.25(\mathrm{~m}, 1 \mathrm{H}), 3.78(\mathrm{~s}, 3 \mathrm{H}), 3.67(\mathrm{~s}, 3 \mathrm{H}) ;{ }^{13} \mathrm{C}\left\{{ }^{1} \mathrm{H}\right\} \operatorname{NMR}(100$ $\left.\mathrm{MHz}, \mathrm{CDCl}_{3}\right) \delta 172.8(\mathrm{~d}, J=7 \mathrm{~Hz}), 159.4,132.8,132.4-131.8(\mathrm{~m}), 131.6,131.0,130.4(\mathrm{~d}, J=5 \mathrm{~Hz}), 128.6-$ $128.2(\mathrm{~m}), 114.1,56.2,55.3,52.8 ;{ }^{31} \mathrm{P}$ NMR $\left(162 \mathrm{MHz}, \mathrm{CDCl}_{3}\right) \delta 23.5$; HRMS (EI) calcd for $\left[\mathrm{C}_{22} \mathrm{H}_{23} \mathrm{NO}_{4} \mathrm{P}\right]^{+}$396.1365, Found 396.1371; Anal. Calcd. for $\mathrm{C}_{22} \mathrm{H}_{22} \mathrm{NO}_{4} \mathrm{P}: \mathrm{C}$ 66.83, H 5.61, N 3.54; Found C 66.89, H 5.81, N 3.63; \%ee by HPLC (Chiralcel OJ Column , 2-propanol:hexanes = 10:90 $(1.0 \mathrm{~mL} / \mathrm{min})$, major isomer $12.0 \mathrm{~min}$, minor isomer $18.2 \mathrm{~min}$ ): $95 \%$ ee.

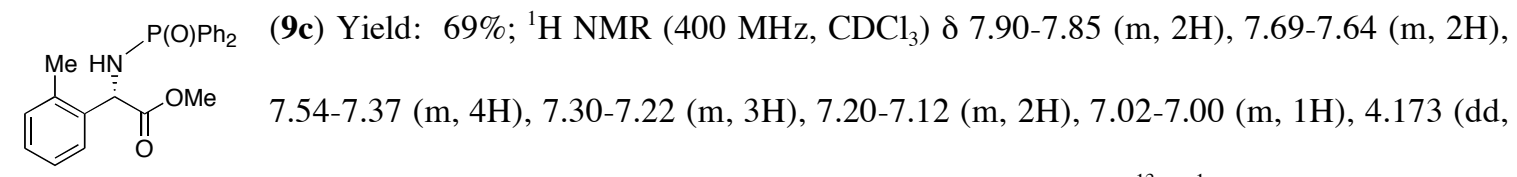
$J=11.6,8.4 \mathrm{~Hz}, 1 \mathrm{H}), 4.39(\mathrm{dd}, J=8.4,8.0 \mathrm{~Hz}, 1 \mathrm{H}), 3.64(\mathrm{~s}, 3 \mathrm{H}), 2.05(\mathrm{~s}, 3 \mathrm{H}) ;{ }^{13} \mathrm{C}\left\{{ }^{1} \mathrm{H}\right\} \mathrm{NMR}(100 \mathrm{MHz}$, $\left.\mathrm{CDCl}_{3}\right) \delta 173.0(\mathrm{~d}, J=6 \mathrm{~Hz}), 159.6,137.1(\mathrm{~d}, J=3 \mathrm{~Hz}), 132.9,132.5-131.7(\mathrm{~m}), 130.7,130.5,128.6-128.0$ (m), 126.6, 126.4, 52.9, 52.8, $19.0 ;{ }^{31} \mathrm{P}$ NMR (162 $\left.\mathrm{MHz}, \mathrm{CDCl}_{3}\right) \delta 23.3$; HRMS (EI) calcd for $\left[\mathrm{C}_{22} \mathrm{H}_{23} \mathrm{NO}_{3} \mathrm{P}\right]^{+}$380.1416, Found 380.1420; \%ee by HPLC (Chiralcel OJ Column , 2-propanol:hexanes = 5:95 (1.0 $\mathrm{mL} / \mathrm{min})$, major isomer $9.9 \mathrm{~min}$, minor isomer $17.9 \mathrm{~min}): 99 \%$ ee. 
(11a) Yield: $71 \% ;{ }^{1} \mathrm{H} \mathrm{NMR}\left(400 \mathrm{MHz}, \mathrm{CDCl}_{3}\right) \delta$ 7.95-7.85 (m, 4H), 7.69-7.62 (m, $7.56-7.37(\mathrm{~m}, 5 \mathrm{H}), 7.32-7.25(\mathrm{~m}, 2 \mathrm{H}), 7.21-7.11(\mathrm{~m}, 3 \mathrm{H}), 6.08(\mathrm{~s}, 1 \mathrm{H}), 3.70-3.59$ $(\mathrm{m}, 1 \mathrm{H}), 3.04-3.00(\mathrm{~m}, 1 \mathrm{H}), 1.87-1.59(\mathrm{~m}, 5 \mathrm{H}), 0.88(\mathrm{t}, J=7.5 \mathrm{~Hz}, 3 \mathrm{H}) ;{ }^{13} \mathrm{C}\left\{{ }^{1} \mathrm{H}\right\} \mathrm{NMR}\left(75 \mathrm{MHz}^{\mathrm{CDCl}} \mathrm{CD}_{3}\right) \delta$ $138.3,137.9,133.0-132.0(\mathrm{~m}), 129.2-128.3(\mathrm{~m}), 127.5,126.6,61.0,29.3(\mathrm{~d}, J=4.5 \mathrm{~Hz}), 13.3,10.9 ;{ }^{31} \mathrm{P}$ NMR (162 MHz, $\left.\mathrm{CDCl}_{3}\right) \delta 22.5$; HRMS (EI) calcd for $\left[\mathrm{C}_{24} \mathrm{H}_{27} \mathrm{NOP}\right]^{+} 375.1830$, Found 376.1833; \%ee by HPLC (Chiralcel OD Column , 2-propanol:hexanes = 15:85 (1.0 mL/min), minor isomer 5.3 min, major isomer $10.9 \mathrm{~min})$ : $99 \%$ ee.

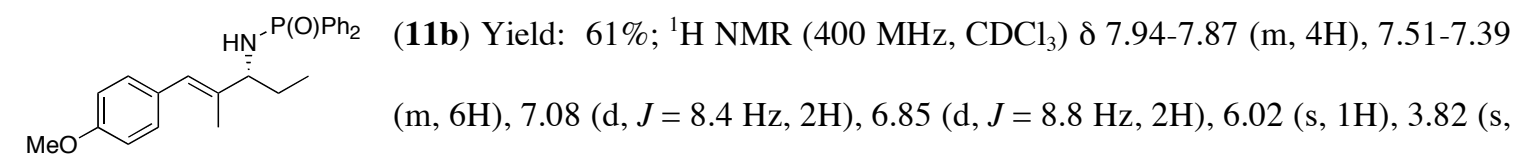
$3 \mathrm{H}), 3.68-3.60(\mathrm{~m}, 1 \mathrm{H}), 3.02-2.98(\mathrm{~m}, 1 \mathrm{H}), 1.80(\mathrm{~s}, 3 \mathrm{H}), 2.75-1.48(\mathrm{~m}, 2 \mathrm{H}), 0.89(\mathrm{t}, J=7.6 \mathrm{~Hz}, 3 \mathrm{H}) ;{ }^{31} \mathrm{P}$ NMR (162 MHz, $\left.\mathrm{CDCl}_{3}\right) \delta 22.4$; HRMS (EI) calcd for $\left[\mathrm{C}_{25} \mathrm{H}_{28} \mathrm{NO}_{2} \mathrm{P}\right]^{+}$405.1858, Found 405.1855; \%ee by HPLC (Chiralcel OD Column , 2-propanol:hexanes = 5:95 (1.0 mL/min), minor isomer 15.0 min, major isomer $18.1 \mathrm{~min})$ : $92 \%$ ee.

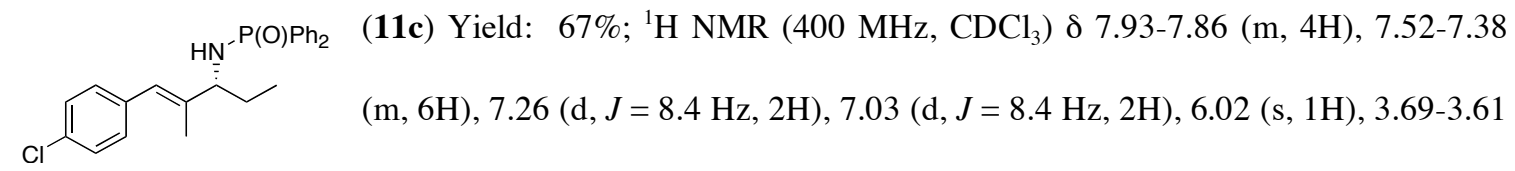
$(\mathrm{m}, 1 \mathrm{H}), 3.03-2.99(\mathrm{~m}, 1 \mathrm{H}), 1.77(\mathrm{~d}, J=1.2 \mathrm{~Hz}, 3 \mathrm{H}), 1.83-1.75(\mathrm{~m}, 1 \mathrm{H}), 1.72-1.63(\mathrm{~m}, 1 \mathrm{H}), 0.90(\mathrm{t}, J=7.6$ $\mathrm{Hz}, 3 \mathrm{H}) ;{ }^{13} \mathrm{C}\left\{{ }^{1} \mathrm{H}\right\} \quad \mathrm{NMR}\left(100 \mathrm{MHz}, \mathrm{CDCl}_{3}\right) \delta 138.7(\mathrm{~d}, J=4.0 \mathrm{~Hz}), 135.9,132.6,132.0-131.7(\mathrm{~m}), 130.1$, 128.5-128.1 (m), 126.1, 60.6, 28.8, 12.9, 10.6; ${ }^{31} \mathrm{P}$ NMR (162 MHz, $\left.\mathrm{CDCl}_{3}\right) \delta 22.2 ; \mathrm{HRMS}$ (EI) calcd for $\left[\mathrm{C}_{24} \mathrm{H}_{25} \mathrm{ClNOP}\right]^{+}$409.1362, Found 409.1363; \%ee by HPLC (Chiralpak AD Column , ethanol:hexanes = 5:95 (1.3 $\mathrm{mL} / \mathrm{min})$, minor isomer $12.2 \mathrm{~min}$, major isomer $13.5 \mathrm{~min}): 97 \%$ ee.

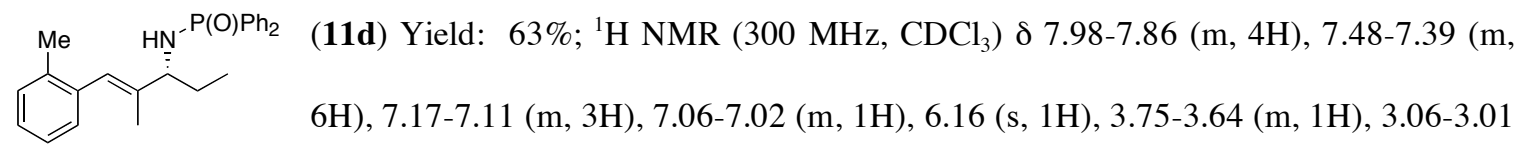
$(\mathrm{m}, 1 \mathrm{H}), 2.15(\mathrm{~s}, 3 \mathrm{H}), 1.92-1.78(\mathrm{~m}, 1 \mathrm{H}), 1.77-1.60(\mathrm{~m}, 1 \mathrm{H}), 1.65(\mathrm{~d}, J=1.2 \mathrm{~Hz}, 3 \mathrm{H}), 0.91(\mathrm{t}, J=9.6 \mathrm{~Hz}$, $3 \mathrm{H}) ;{ }^{13} \mathrm{C}\left\{{ }^{1} \mathrm{H}\right\} \quad \mathrm{NMR}\left(75 \mathrm{MHz}, \mathrm{CDCl}_{3}\right) \delta 138.4(\mathrm{~d}, J=5.3 \mathrm{~Hz}), 137.1 .136 .7,132.9-128.6(\mathrm{~m}), 127.0,126.4$. 125.5, 60.5, $29.4(\mathrm{~d}, J=4.5 \mathrm{~Hz}), 20.2,13.1,10.9 ;{ }^{31} \mathrm{P}$ NMR (122 MHz, $\left.\mathrm{CDCl}_{3}\right) \delta 22.3$; HRMS (EI) calcd for 
$\left[\mathrm{C}_{25} \mathrm{H}_{28} \mathrm{NOP}\right]^{+}$389.1909, Found 389.1900; \%ee by HPLC (Chiralpak AD Column , 2-propanol:hexanes = 10:90 (1.0 $\mathrm{mL} / \mathrm{min})$, minor isomer $10.1 \mathrm{~min}$, major isomer $13.6 \mathrm{~min}): 74 \%$ ee.

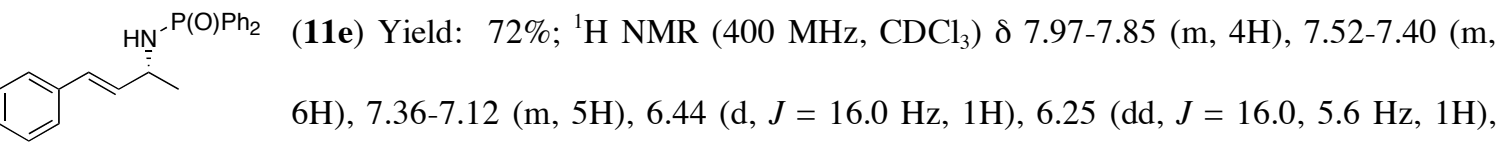
4.04-3.93 (m, 1H), 2.99-2.95 (m, 1H), $1.44(\mathrm{~d}, J=6.8 \mathrm{~Hz}, 3 \mathrm{H}) ;{ }^{13} \mathrm{C}\left\{{ }^{1} \mathrm{H}\right\} \quad \mathrm{NMR}\left(100 \mathrm{MHz}, \mathrm{CDCl}_{3}\right) \delta 136.7$, 133.7-131.8, 128.9, 128.6-128.3 (m), 127.5, 126.4, 49.0, $24.1(\mathrm{~d}, J=4.0 \mathrm{~Hz}) ;{ }^{31} \mathrm{P}$ NMR $\left(122 \mathrm{MHz}, \mathrm{CDCl}_{3}\right)$ $\delta$ 22.5; HRMS (EI) calcd for $\left[\mathrm{C}_{22} \mathrm{H}_{22} \mathrm{NOP}\right]^{+} 347.1439$, Found 347.1444; \%ee by HPLC (Chiralcel OD Column , 2-propanol:hexanes = 15:85 $(1.0 \mathrm{~mL} / \mathrm{min})$, minor isomer $7.3 \mathrm{~min}$, major isomer $8.5 \mathrm{~min}): 75 \%$ ee.

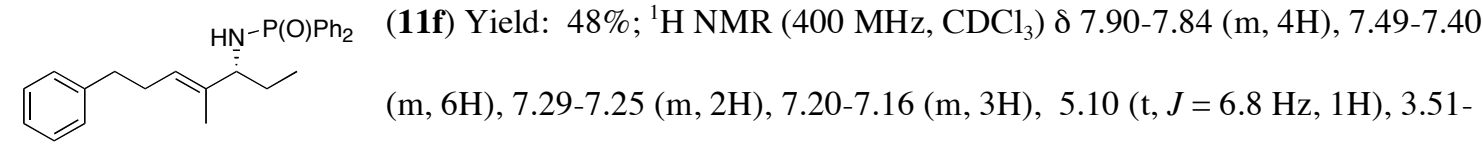

3.3.42 (m, 1H), 2.89-2.83 (m, 1H), 2.59-2.53 (m, 2H) 2.28-2.22 (m, 2H), 1.72-1.64 (m, 1H), 1.57-1.49 (m, 1H), $1.52(\mathrm{~s}, 3 \mathrm{H}), 0.75(\mathrm{t}, J=7.6 \mathrm{~Hz}, 3 \mathrm{H}) ;{ }^{13} \mathrm{C}\left\{{ }^{1} \mathrm{H}\right\}$ NMR $\left(100 \mathrm{MHz}, \mathrm{CDCl}_{3}\right) \delta 132.6-131.7(\mathrm{~m}), 128.5-$ $128.1(\mathrm{~m}), 126.4,125.7,60.2,35.6,29.4,28.7(\mathrm{~d}, J=4.0 \mathrm{~Hz}) 11.4,10.4 ;{ }^{31} \mathrm{P} \mathrm{NMR}\left(122 \mathrm{MHz}, \mathrm{CDCl}_{3}\right) \delta$ 22.0; HRMS (EI) calcd for $\left[\mathrm{C}_{26} \mathrm{H}_{30} \mathrm{NOP}\right]^{+}$403.2065, Found 403.2068; \% ee by HPLC (Chiralcel OD Column , 2-propanol:hexanes = 5:95 (1.0 mL/min), minor isomer $12.7 \mathrm{~min}$, major isomer $14.7 \mathrm{~min}): 83 \%$ ee.

${ }^{1}$ Müller, D.; Umbricht, G.; Weber, B.; Pfaltz, A. Helv. Chim. Acta 1991, 74, 232; Hall, J.; Lehn, J.-M.; De Cian, A.; Fischer, J. Helv. Chim. Acta 1991, 74, 1.

${ }^{2}$ Kawasaki, K.; Katsuki, T. Tetrahedron 1997, 53 (18), 6337; Tang, T. P.; Volkman, S. K.; Ellman, J. A. J. Org. Chem. 2001, 66, 8772.

${ }^{3}$ Corey, E. J.; Wang, Z. Tetrahedron Lett. 1993, 34 (25), 4001.

${ }^{4}$ Sherry, B. D.; Loy, R. N.; Toste, F. D. J. Am. Chem. Soc. 2004, 126 (14), 4510.

${ }^{5}$ Masumoto, S.; Usuda, H.; Suzuki, M.; Kanai, M.; Shibaski, M. J. Am. Chem. Soc 2003, 125, 5634.

${ }^{6}$ Yamada, K.; Harwood, S.; Groger, H.; Shibasaki Angew. Chem. Int. Ed 1999, 38 (23), 3504.

${ }^{7}$ Yamada, T.; Nagata, T.; Sugi, K.; Yorozu, K.; Ikeno, T.; Ohtsuka, Y.; Miyazaki, D.; Mukaiyama, T. Chem. Eur. J 2003, $9,4485$. 


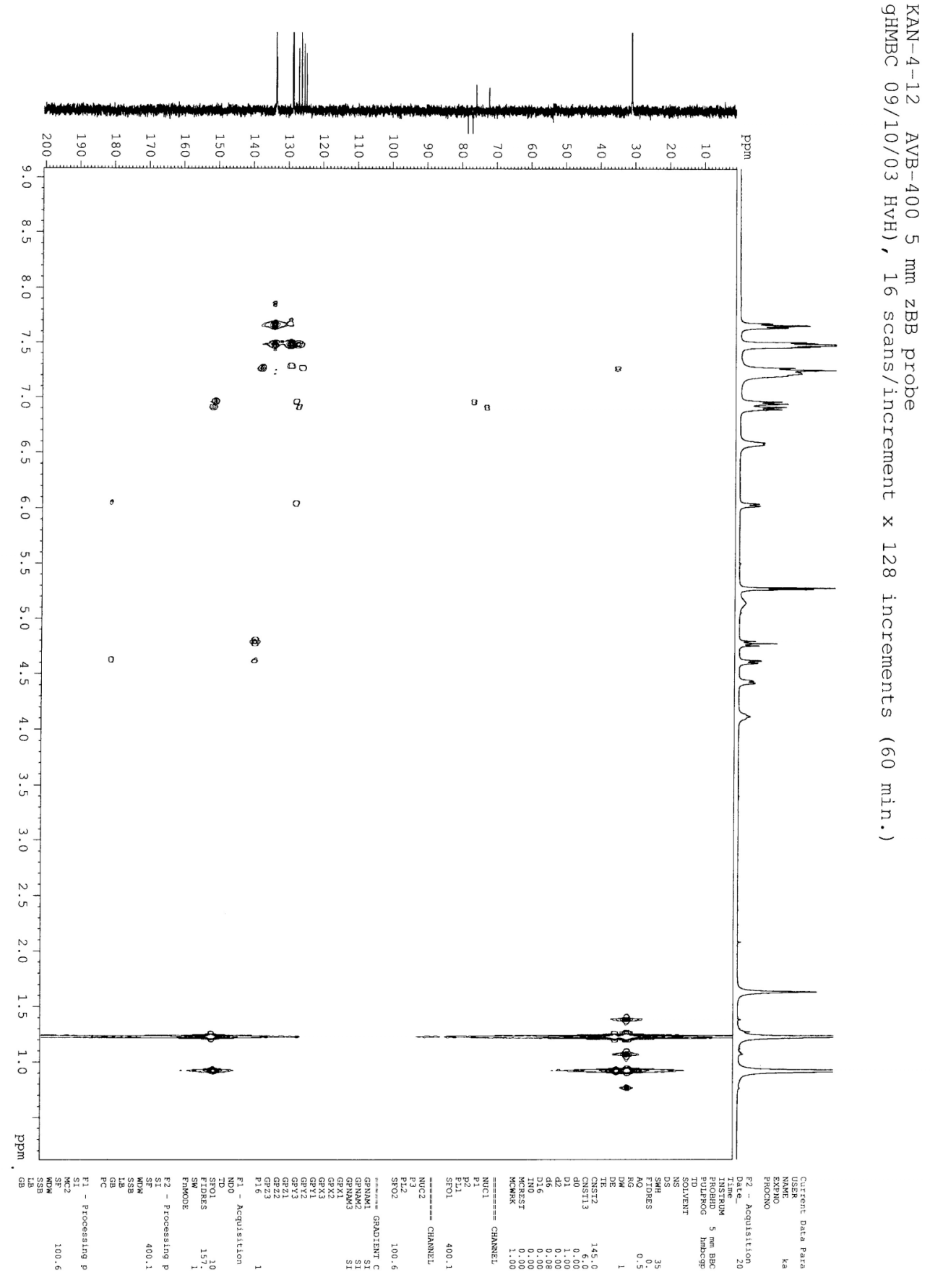




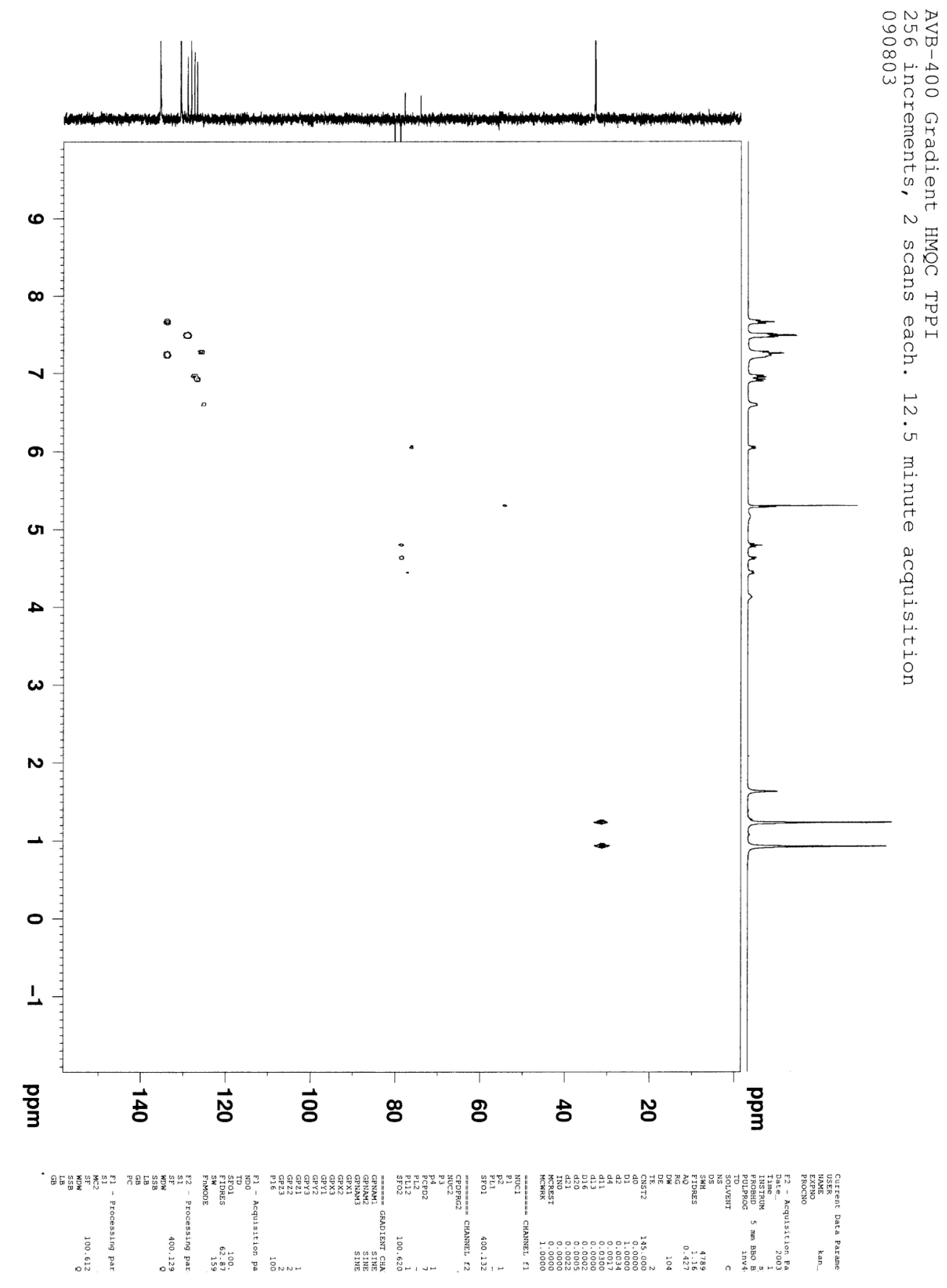

